\title{
Anomalous Connection of Umbilical Vessels to the Left Ventricle: Case Report
}

\author{
Yo Han Bae, M.D. ${ }^{1}$, Woo Sung Jang, M.D., Ph.D. ${ }^{1}$, Hee Joung Choi, M.D. ${ }^{2}$, So Young Shin, M.D. ${ }^{2}$ \\ Departments of 'Thoracic and Cardiovascular Surgery and 'Pediatrics, Keimyung University Dongsan Medical Center, Keimyung University College of Medicine, \\ Daegu, Korea
}

\section{ARTICLE INFO}

Received September 3, 2020

Revised October 12, 2020

Accepted October 28, 2020

Corresponding author

Woo Sung Jang

Tel 82-53-258-7966

Fax 82-53-258-4783

E-mail whiteuri@dsmc.or.kr

ORCID

https://orcid.org/0000-0003-1576-9472
The anomalous connection of umbilical vessels to the heart is rare and has not yet been reported in the international scientific literature. Herein, we report the case of a newborn who was diagnosed with an anomalous connection of the umbilical vessels to the left ventricle. These anomalous vessels were functionally open for 2 weeks, and cellulitis was present in the area of the blood vessels connected to the skin. We performed division of these abnormal vessels and removal of the skin lesion.

Keywords: Neonate, Umbilical vessels, Case report

\section{Case report}

A female infant weighing 3,500 g was born via emergency cesarean section at 39 weeks of gestation due to intrauterine fetal distress. The mother had well-controlled gestational diabetes mellitus and hyperthyroidism. On prenatal ultrasound scans, the fetus exhibited mesocardia. At birth, the infant's vital saturation was $80 \%$ in room air, and her heart rate was 140 beats/min. For these reasons, she was transferred to the neonatal intensive care unit. After conservative management for hypoxia, the patient's saturation recovered.

During the physical examination, an additional anomalous umbilical cord originating from the xiphoid process and extending to the umbilicus on the skin was observed (Fig. 1). Chest computed tomography (CT) and chest wall Doppler ultrasonography were performed to confirm the insertion site of the anomalous umbilical cord. Chest CT showed mesocardia, a focal pericardial defect on the mid-inferior aspect of the cardiac apex, and 2 tiny $(3 \mathrm{~mm}$ each) enhancing umbilical vessels extending from the left ventricle (LV) (Fig. 2). Chest wall Doppler ultrasonography showed a vascular sac originating from the LV myocardial wall (Fig. 3). In addition, a 2-mm perimembranous ventricular septal defect and a 3-mm secundum atrial septal de-

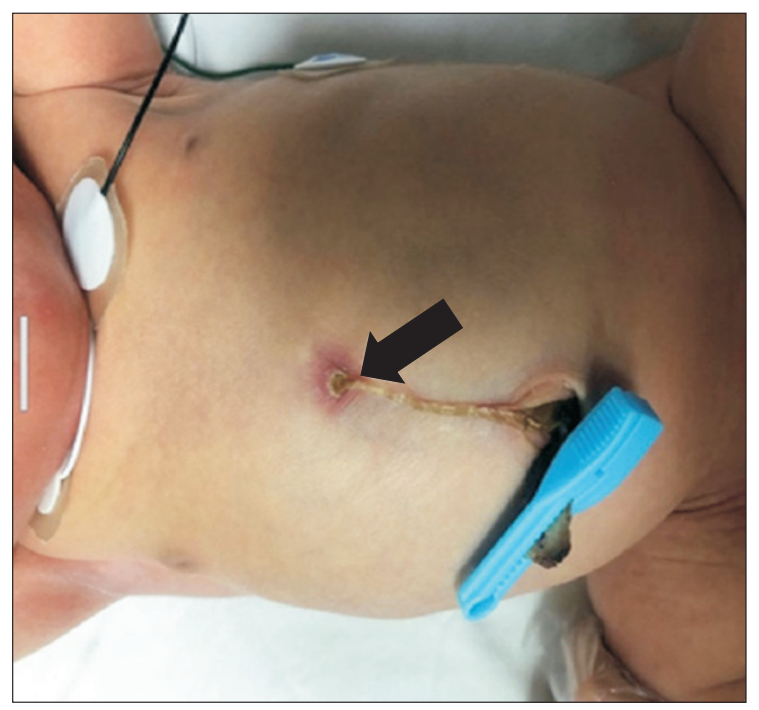

Fig. 1. Gross features of the anomalous umbilical vessels (arrow) originating from the xiphoid process. Written informed consent for publication of this image was obtained from the patient's family.

fect were observed via echocardiography.

No complications related to the anomalous umbilical cord were observed. However, 4 days after birth, redness with a local sensation of heat was observed at the insertion site of the anomalous umbilical cord around the xiphoid 
process. Accompanying leukocytosis and an elevated C-reactive protein level suggested cellulitis.

To treat the cellulitis, empirical antibiotics were administered, and a povidone-iodine dressing was applied daily. After the cellulitis was controlled, 13 days after birth, follow-up CT and chest wall Doppler scans were performed and showed no interval change in the abnormal vessels. Thus, surgical treatment was planned. For the division of the anomalous umbilical cord, we approached with a subxiphoid incision around the cord insertion site. After the abnormal umbilical vessels were exposed, they were divided as proximal to the heart as possible using a clip and a black silk tie (Fig. 4). The sac connected to the skin was removed, and the wound was closed. After surgery, the wound exhibited good recovery, and the patient was discharged without any other complications. At the 11-month outpatient follow-up visit, no additional abnormal umbilical vessels were observed. The patient's family provided written informed consent for the publication of clinical de-

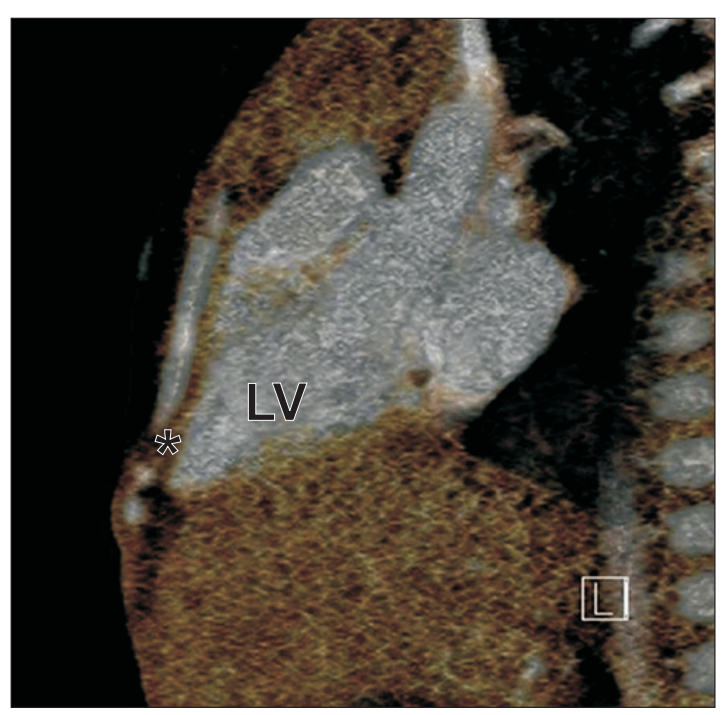

Fig. 2. Umbilical vessels connected to the left ventricle (LV) in chest computed tomography. The asterisk $(*)$ indicates the umbilical vessel emerging from the LV. tails and images.

\section{Discussion}

The umbilical cord forms a stable connection between a healthy fetus and the placenta at the level of the feto-maternal interface. However, abnormalities of the umbilical cord related to morphology, placental insertion, the number of vessels, and other factors can influence perinatal outcomes and may be associated with other fetal anomalies. Although the pathophysiology of the anomaly in this case was unclear, we suspected several genetic diseases. Ectopia cordis is a rare genetic defect whereby the chest wall does not fuse correctly in utero [1]. Another relevant anomaly is the pentalogy of Cantrell, which is characterized by a combination of midline birth defects that can involve the sternum, or breastbone; the diaphragm, or muscle that separates the chest cavity from the abdomen and aids in breathing; the pericardium, or thin membrane that

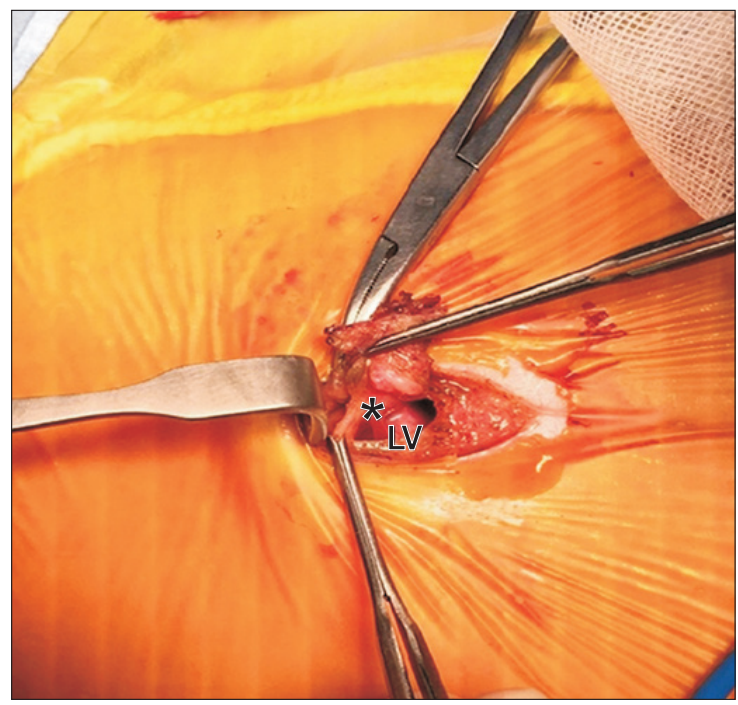

Fig. 4. Surgical view of anomalous umbilical vessels connected to the left ventricle (LV). The asterisk $(*)$ indicates the umbilical vessel emerging from the LV.
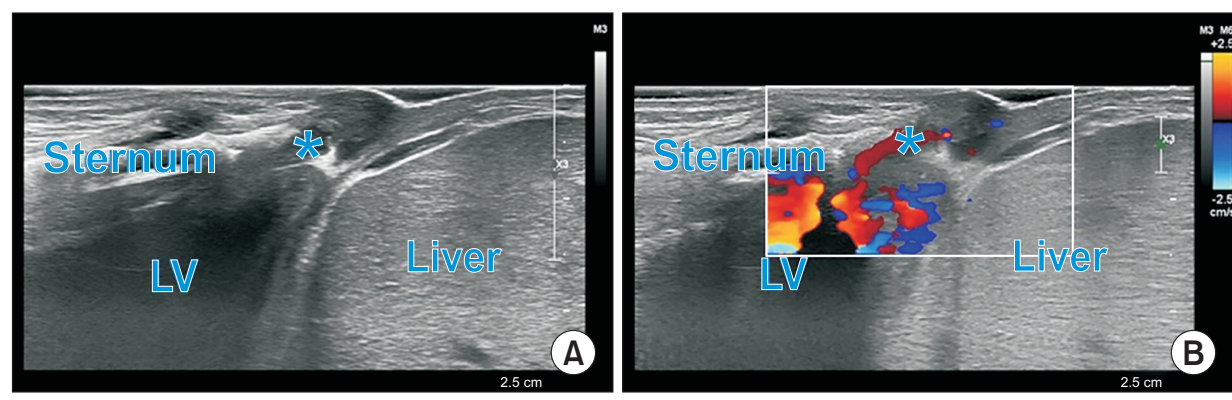

Fig. 3. (A, B) Blood flow between the left ventricle (LV) and the umbilical vessels as visualized with chest wall Doppler ultrasonography. The asterisk $(*)$ indicates the umbilical vessel emerging from the LV. 
lines the heart; the abdominal wall; and congenital heart defects, such as ventricular septal defects $[2,3]$.

This case was thought to be a mild form of ectopia cordis or pentalogy of Cantrell, given the positioning of the apex of the heart toward the chest wall, the diaphragm surface defect of the pericardium, and the accompanying congenital heart anomaly. Pentalogy of Cantrell has been suggested to be caused by the failure of proper midline mesoderm maturation and ventral body wall formation during embryonic development [1].

In general, functional closure of the umbilical vessels occurs within the first minutes of life [4]. Thus, one option may be to wait until the blood flow of the umbilical vessels is blocked naturally. However, in the present case, we observed no interval change in the abnormal umbilical vessels during the 13 days prior to the follow-up CT scan. The clinical course of cases involving abnormal umbilical vessels connected to the LV is unknown, and aneurysm formation in the umbilical vessels is possible when this condition is not treated. In addition, repeated infections of the cord insertion site are expected to spread into the LV. For these reasons, in the present case, we conducted surgical division. As no previous reports have been published on abnormal umbilical vessels connected to the LV, we report this rare case.

\section{Conflict of interest}

No potential conflict of interest relevant to this article was reported.

\section{ORCID}

Yo Han Bae: https://orcid.org/0000-0001-7054-1898

Woo Sung Jang: https://orcid.org/0000-0003-1576-9472

Hee Joung Choi: https://orcid.org/0000-0002-7119-4194

So Young Shin: https://orcid.org/0000-0001-8328-1087

\section{References}

1. Pius S, Abubakar Ibrahim H, Bello M, Bashir Tahir M. Complete ectopia cordis: a case report and literature review. Case Rep Pediatr 2017;2017:1858621.

2. Smigiel R, Jakubiak A, Lombardi MP, et al. Co-occurrence of severe Goltz-Gorlin syndrome and pentalogy of Cantrell: case report and review of the literature. Am J Med Genet A 2011;155A:1102-5.

3. Singh N, Bera ML, Sachdev MS, Aggarwal N, Joshi R, Kohli V. Pentalogy of Cantrell with left ventricular diverticulum: a case report and review of literature. Congenit Heart Dis 2010;5:454-7.

4. Yao AC, Lind J, Lu T. Closure of the human umbilical artery: a physiological demonstration of Burton's theory. Eur J Obstet Gynecol Reprod Biol 1977;7:365-8. 